\begin{tabular}{|c|l|}
\hline Title & Motion of a vortex sheet on a sphere with pole vortices \\
\hline Author(s) & Sakajo, Takashi \\
\hline Citation & $\begin{array}{l}\text { Physics of Fluids, 16(3), 717-727 } \\
\text { https://doi.org/10.1063/1644148 }\end{array}$ \\
\hline Issue Date & 2004-02-04 \\
\hline Doc URL & http://hdl.handle.net/2115/5433 \\
\hline Rights & Copyright $\odot 2004$ A merican Institute of Physics \\
\hline Type & article \\
\hline File Information & PF16-3.pdf \\
\hline
\end{tabular}

Instructions for use 


\title{
Motion of a vortex sheet on a sphere with pole vortices
}

\author{
Takashi Sakajo ${ }^{\text {a) }}$ \\ Department of Mathematics, Hokkaido University, Sapporo, Hokkaido 060-8610, Japan
}

(Received 28 May 2003; accepted 1 December 2003; published online 4 February 2004)

\begin{abstract}
We consider the motion of a vortex sheet on the surface of a unit sphere in the presence of point vortices fixed on north and south poles. Analytic and numerical research revealed that a vortex sheet in two-dimensional space has the following three properties. First, the vortex sheet is linearly unstable due to Kelvin-Helmholtz instability. Second, the curvature of the vortex sheet diverges in finite time. Last, the vortex sheet evolves into a rolling-up doubly branched spiral, when the equation of motion is regularized by the vortex method. The purpose of this paper is to investigate how the curvature of the sphere and the presence of the pole vortices affect these three properties mathematically and numerically. We show that some low spectra of disturbance become linearly stable due to the pole vortices and thus the singularity formation tends to be delayed. On the other hand, however, the vortex sheet, which is regularized by the vortex method, acquires complex structure of many rolling-up spirals. (C) 2004 American Institute of Physics.
\end{abstract}

[DOI: $10.1063 / 1.1644148]$

\section{INTRODUCTION}

In real fluid flows, we often observe a band-like region in which the velocity field changes rapidly and thus the vorticity highly concentrates. The region induces a shear flow, which is one of the basic research objects in the field of fluid dynamics. A vortex sheet is a simple model of the shear flow, if the thickness of the high vorticity region is infinitely thin; in other words, it is defined as a discontinuous surface of the velocity field in incompressible and inviscid flow.

Vortex sheets in two-dimensional (2D) space have been investigated mathematically as well as numerically for several decades. We summarize some basic properties of the 2D vortex sheet obtained so far. See a review in Saffman ${ }^{1}$ for the details. A 2D vortex sheet is represented by a complexvalued function

$$
z(\Gamma, t)=x(\Gamma, t)+i y(\Gamma, t), \quad-\infty<\Gamma<\infty,
$$

where $\Gamma$ is Lagrangian parameter along the sheet and $t$ is time. Then, the motion of the vortex sheet is governed by the Birkhoff-Rott equation ${ }^{2}$

$$
\frac{\partial \bar{z}}{\partial t}(\Gamma, t)=\frac{1}{2 \pi i} \mathrm{PV} \int_{-\infty}^{\infty} \frac{d \Gamma^{\prime}}{z(\Gamma, t)-z\left(\Gamma^{\prime}, t\right)},
$$

in which the integral is Cauchy's principal value integral and $\bar{z}$ denotes the complex conjugate of $z$.

First, the vortex sheet is linearly unstable. A flat vortex sheet $z(\Gamma, t)=\Gamma$ is a stationary solution of the Birkhoff-Rott equation. When we add a small disturbance to the steady state, that is to say

$$
z(\Gamma, t)=\Gamma+\sum_{n=-\infty}^{\infty} a_{n}(t) \exp (i n \Gamma)
$$

a) Telephone: +81-11-706-4660; fax: +81-11-727-3705; electronic mail: sakajo@math.sci.hokudai.ac.jp then linearization of the Birkhoff-Rott equation provides us with linear equations for the spectra $a_{n}(t)$ and consequently we obtain

$$
\left|a_{n}(t)\right| \sim\left|a_{n}(0)\right| \exp \left(\frac{1}{2}|n| t\right)
$$

for $t \ll 1$. It indicates that the spectrum $a_{n}(t)$ grows exponentially in time at a rate proportional to the wave number $n$. This instability is known as Kelvin-Helmholtz instability. Hence, we notice that the Birkhoff-Rott equation is ill-posed in the sense of Hadamard.

The next result is concerned with the existence of the solution to the Birkhoff-Rott equation. Moore ${ }^{3}$ considered the spectral form of solution (1) with an initial condition

$$
z(\Gamma, t)=\Gamma+i \epsilon \sin \Gamma \text {. }
$$

Then, asymptotic analysis shows that the spectrum $a_{n}(t)$ has the following asymptotic form:

$$
\left|a_{n}(t)\right| \sim C n^{-5 / 2} \exp \left(n\left(1+\frac{1}{2} t+\frac{1}{4} \log \epsilon t\right)\right),
$$

for $t \gg 1$, where $C$ is a constant independent of $n$. Therefore, if $t_{c}$ satisfies

$$
1+\frac{1}{2} t_{c}+\frac{1}{4} \log \epsilon t_{c}=0,
$$

then the second derivative of the solution, in other words the curvature of the vortex sheet, diverges at $t_{c}$, since $\left|a_{n}(t)\right|$ is asymptotically similar to $C n^{-5 / 2}$, as $t \rightarrow t_{c}$. It indicates that the solution of the Birkhoff-Rott equation loses its analyticity in finite time. Direct numerical computations of the Birkhoff-Rott equation ${ }^{4-6}$ followed Moore and supported his asymptotic result.

Finally, long time evolution of the vortex sheet is studied numerically. Because of the singularity formation, we are unable to compute the Birkhoff-Rott equation beyond the critical time. On the other hand, however, introducing an artificial parameter $\sigma$, $\mathrm{Krasny}^{7}$ considered the following regularized equation: 


$$
\frac{\partial \bar{z}}{\partial t}=\frac{1}{2 \pi i} \int_{-\infty}^{\infty} K_{\sigma}\left(z(\Gamma, t), z\left(\Gamma^{\prime}, t\right)\right) d \Gamma^{\prime},
$$

where

$$
K_{\sigma}\left(z(\Gamma, t), z\left(\Gamma^{\prime}, t\right)\right)=\frac{\bar{z}(\Gamma, t)-\bar{z}\left(\Gamma^{\prime}, t\right)}{\left|z(\Gamma, t)-z\left(\Gamma^{\prime}, t\right)\right|^{2}+\sigma^{2}} .
$$

When $\sigma=0$, the equation is equivalent to the Birkhoff-Rott equation. This regularization method is called the vortex method. Caflisch and Lowengrub ${ }^{8}$ have proven that the solution of the regularized equation converges uniformly to that of the Birkhoff-Rott equation as $\sigma \rightarrow 0$, as long as both solutions are smooth. After the singularity formation, we have no such strong convergence result. However, Majda ${ }^{9}$ remarked that the regularized equation converges to the Birkhoff-Rott equation in a weak sense, even if the singularity appears. On the other hand, some numerical simulations of the regularized equation indicated that the vortex method gives a physically convincing approximation for incompressible flows with small viscosity. ${ }^{7,10-14}$ In fact, Krasny $^{7}$ showed that the regularized 2D vortex sheet rolls up into a doubly branched spiral, which is a familiar phenomenon observed in real fluid flows.

Vortex dynamics on a sphere plays an important role in understanding basic flows on Earth. There has been much research on $N$ point vortices (see references in Newton's book $)^{15}$ and vortex layers with constant vorticity ${ }^{16,17}$ on a sphere. In the present paper, we consider a vortex sheet on the surface of a sphere. This is a model of shear layers on the Earth when we neglect the effect of rotation. Besides, we assume that two vortex points are fixed at the north and south poles. The purpose of the study is to investigate how the curvature of the sphere and the pole vortices affect the three properties obtained in the study of 2D vortex sheets: linear stability, singularity formation, and structure of rolling-up spirals.

This paper consists of six sections. In Sec. II, we derive a governing equation for a vortex sheet on a unit sphere in the presence of the two pole point vortices. In Sec. III, we study the linear stability of the vortex sheet. Then, we deal with the singularity formation in Sec. IV. In Sec. V, we compute long time evolution of the regularized vortex sheet and study how the presence of the pole vortices changes the spiral structure of the vortex sheet. In Sec. VI, we conclude the results and compare them with evolution of the band of constant vorticity on a sphere studied by Dritschel and Polvani. ${ }^{16}$

\section{GOVERNING EQUATIONS}

Suppose that the position of a vortex sheet on a unit sphere with constant vorticity density is represented by $(\theta(\alpha, t), \varphi(\alpha, t))$ in the spherical coordinates, where $\alpha$ $\in[0,2 \pi)$ is Lagrangian parameter along the sheet and $t$ is time. Then, starting with the equations of motion for $N$ point vortices, we derive the equation for the vortex sheet by taking the limit $N \rightarrow \infty$.

Now, we discretize the vortex sheet by $N$ segments and put a point vortex with strength $h=2 \pi / N$ at $\left(\theta_{i}(t), \varphi_{i}(t)\right)$ $=(\theta(i h, t), \varphi(i h, t))$ for $i=1, \ldots, N$. Then, Kimura and Okamoto $^{18}$ showed that the $N$ point vortices induce the following velocity field $\left(v_{\theta}^{N}, v_{\varphi}^{N}\right)$ at the position of $m$ th point vortex, $\left(\theta_{m}, \varphi_{m}\right)$ :

$$
\begin{aligned}
v_{\theta}^{N}\left(\theta_{m}, \varphi_{m}\right)= & -\frac{h}{4 \pi} \sum_{i \neq m}^{N} \frac{\sin \theta_{i} \sin \left(\varphi_{m}-\varphi_{i}\right)}{1-\cos \gamma_{m i}}, \\
v_{\varphi}^{N}\left(\theta_{m}, \varphi_{m}\right)= & -\frac{h}{4 \pi} \sum_{i \neq m}^{N} \\
& \times \frac{\cos \theta_{m} \sin \theta_{i} \cos \left(\varphi_{m}-\varphi_{i}\right)-\sin \theta_{m} \cos \theta_{i}}{\sin \theta_{m}\left(1-\cos \gamma_{m i}\right)},
\end{aligned}
$$

where $\gamma_{m i}$ denotes the central angle between two points $\left(\theta_{i}, \varphi_{i}\right)$ and $\left(\theta_{m}, \varphi_{m}\right)$, and

$$
\cos \gamma_{m i}=\cos \theta_{m} \cos \theta_{i}+\sin \theta_{m} \sin \theta_{i} \cos \left(\varphi_{m}-\varphi_{i}\right) .
$$

As $N \rightarrow \infty$, the discrete velocity fields (3) and (4) converge to the following Cauchy's principal value integrals, which give the velocity field at the position of the vortex sheet $(\theta(\alpha, t), \varphi(\alpha, t))$ :

$$
\begin{aligned}
& v_{\theta}(\theta(\alpha, t), \varphi(\alpha, t))=-\frac{1}{4 \pi} \mathrm{PV} \int_{0}^{2 \pi} \frac{\sin \theta^{\prime} \sin \left(\varphi-\varphi^{\prime}\right)}{1-\cos \gamma} d \alpha^{\prime}, \\
& v_{\varphi}(\theta(\alpha, t), \varphi(\alpha, t))=-\frac{1}{4 \pi} \mathrm{PV} \int_{0}^{2 \pi} \frac{\cos \theta \sin \theta^{\prime} \cos \left(\varphi-\varphi^{\prime}\right)-\sin \theta \cos \theta^{\prime}}{\sin \theta(1-\cos \gamma)} d \alpha^{\prime},
\end{aligned}
$$

in which $\cos \gamma=\cos \theta \cos \theta^{\prime}+\sin \theta \sin \theta^{\prime} \cos \left(\varphi-\varphi^{\prime}\right), \quad \theta$ $=\theta(\alpha, t), \theta^{\prime}=\theta\left(\alpha^{\prime}, t\right)$ and so on.

Furthermore, we introduce two point vortices fixed at the both poles of the sphere for generality. Let $\Gamma_{1}$ and $\Gamma_{2}$ denote the strengths of the point vortices at the north and the south poles, respectively. Since the pole vortices are fixed at $\theta$ $=0$ and $\pi$, the vortex sheet is convected by their inducing flow

$$
\left(v_{\theta}, v_{\varphi}\right)=\left(0, \frac{1}{4 \pi}\left(\frac{\Gamma_{1}}{1-\cos \theta}-\frac{\Gamma_{2}}{1+\cos \theta}\right)\right) .
$$

Hence, we obtain the equation of motion for the single vortex sheet on the unit sphere with the two pole vortices

$\theta_{t}=-\frac{1}{4 \pi} \mathrm{PV} \int_{0}^{2 \pi} \frac{\sin \theta^{\prime} \sin \left(\varphi-\varphi^{\prime}\right)}{1-\cos \gamma} d \alpha^{\prime}$, 


$$
\begin{aligned}
\varphi_{t}= & -\frac{1}{4 \pi} \\
& \times \mathrm{PV} \int_{0}^{2 \pi \cos \theta \sin \theta^{\prime} \cos \left(\varphi-\varphi^{\prime}\right)-\sin \theta \cos \theta^{\prime}} \frac{\sin \theta(1-\cos \gamma)}{4 \pi} d \alpha^{\prime} \\
& +\frac{1}{4 \pi}\left(\frac{\Gamma_{1}}{1-\cos \theta}-\frac{\Gamma_{2}}{1+\cos \theta}\right)
\end{aligned}
$$

\section{LINEAR STABILITY ANALYSIS}

Suppose that the vortex sheet is identical to a line of latitude, namely $\theta(\alpha, t)=\theta_{0}$ and $\varphi(\alpha, t)=\alpha$, then the righthand sides of (7) and (8) become

$$
\begin{aligned}
& \theta_{t}=-\frac{1}{4 \pi \sin \theta_{0}} \mathrm{PV} \int_{0}^{2 \pi} \frac{\sin \left(\alpha-\alpha^{\prime}\right)}{1-\cos \left(\alpha-\alpha^{\prime}\right)} d \alpha^{\prime}=0, \\
& \varphi_{t}=\frac{\cos \theta_{0}}{2 \sin ^{2} \theta_{0}}+\frac{1}{4 \pi}\left(\frac{\Gamma_{1}}{1-\cos \theta_{0}}-\frac{\Gamma_{2}}{1+\cos \theta_{0}}\right) \equiv V_{0} .
\end{aligned}
$$

Hence, the vortex sheet $\theta=\theta_{0}$ and $\varphi=\alpha+V_{0} t$ is a steady solution rotating with the constant speed $V_{0}$. We study the linear stability of this solution.
Assume that the solution is disturbed slightly, that is to say

$$
\begin{aligned}
& \theta(\alpha, t)=\theta_{0}+\epsilon_{\theta}(\alpha, t), \\
& \varphi(\alpha, t)=\alpha+V_{0} t+\epsilon_{\varphi}(\alpha, t) .
\end{aligned}
$$

Then, we expand Eqs. (7) and (8) in terms of $\epsilon_{\theta}, \epsilon_{\theta}^{\prime}, \epsilon_{\varphi}$, and $\epsilon_{\varphi}^{\prime}$. The denominators of the integrand become

$$
\begin{aligned}
\frac{1}{1-\cos \gamma}= & \frac{1}{\sin ^{2} \theta_{0}\left(1-\cos \left(\alpha-\alpha^{\prime}\right)\right)} \\
& \times\left(1-\left(\epsilon_{\theta}+\epsilon_{\theta}^{\prime}\right) \frac{\cos \theta_{0}}{\sin \theta_{0}}-\left(\epsilon_{\varphi}-\epsilon_{\varphi}^{\prime}\right)\right. \\
& \left.\times \frac{\sin \left(\alpha-\alpha^{\prime}\right)}{1-\cos \left(\alpha-\alpha^{\prime}\right)}\right)+O\left(\epsilon^{2}\right)
\end{aligned}
$$

and

$$
\frac{1}{\sin \left(\theta_{0}+\epsilon_{\theta}\right)}=\frac{1}{\sin \theta_{0}}-\epsilon_{\theta} \frac{\cos \theta_{0}}{\sin ^{2} \theta_{0}}+O\left(\epsilon^{2}\right) .
$$

On the other hand, the numerators in the integrand are expanded like

$$
\begin{aligned}
\sin \theta^{\prime} \sin \left(\varphi-\varphi^{\prime}\right)= & \sin \theta_{0} \sin \left(\alpha-\alpha^{\prime}\right)+\epsilon_{\theta}^{\prime} \cos \theta_{0} \sin \left(\alpha-\alpha^{\prime}\right) \\
& +\left(\epsilon_{\varphi}-\epsilon_{\varphi}^{\prime}\right) \sin \theta_{0} \cos \left(\alpha-\alpha^{\prime}\right)+O\left(\epsilon^{2}\right),
\end{aligned}
$$

and

$$
\begin{aligned}
\cos \theta \sin \theta^{\prime} \cos \left(\varphi-\varphi^{\prime}\right)-\sin \theta \cos \theta^{\prime}= & -\sin \theta_{0} \cos \theta_{0}\left(1-\cos \left(\alpha-\alpha^{\prime}\right)\right)-\epsilon_{\theta}\left(\cos ^{2} \theta_{0}+\sin ^{2} \theta_{0} \cos \left(\alpha-\alpha^{\prime}\right)\right) \\
& +\epsilon_{\theta}^{\prime}\left(\cos ^{2} \theta_{0} \cos \left(\alpha-\alpha^{\prime}\right)+\sin ^{2} \theta_{0}\right)-\left(\epsilon_{\varphi}-\epsilon_{\varphi}^{\prime}\right) \sin \theta_{0} \cos \theta_{0} \sin \left(\alpha-\alpha^{\prime}\right)+O\left(\epsilon^{2}\right) .
\end{aligned}
$$

From these expressions, we calculate the constant term and the linear terms for $\epsilon_{\theta}, \epsilon_{\theta}^{\prime}$ and $\epsilon_{\varphi}-\epsilon_{\varphi}^{\prime}$ of the integrand in (7) as follows:

$$
\begin{aligned}
& O(1): \frac{\sin \left(\alpha-\alpha^{\prime}\right)}{\sin \theta_{0}\left(1-\cos \left(\alpha-\alpha^{\prime}\right)\right)}, \\
& O\left(\epsilon_{\theta}\right):-\frac{\cos \theta_{0} \sin \left(\alpha-\alpha^{\prime}\right)}{\sin ^{2} \theta_{0}\left(1-\cos \left(\alpha-\alpha^{\prime}\right)\right)}, \\
& O\left(\epsilon_{\theta}^{\prime}\right): 0,
\end{aligned}
$$

and

$$
O\left(\epsilon_{\varphi}-\epsilon_{\varphi}^{\prime}\right):-\frac{1}{\sin \theta_{0}\left(1-\cos \left(\alpha-\alpha^{\prime}\right)\right)}
$$

As for the integrand in Eq. (8), we have

$$
\begin{aligned}
& O(1):-\frac{\cos \theta_{0}}{\sin ^{2} \theta_{0}}, \\
& O\left(\epsilon_{\theta}\right): \frac{1+\cos ^{2} \theta_{0}}{\sin ^{3} \theta_{0}}-\frac{1}{\sin ^{3} \theta_{0}\left(1-\cos \left(\alpha-\alpha^{\prime}\right)\right)},
\end{aligned}
$$

$$
O\left(\epsilon_{\theta}^{\prime}\right): \frac{1}{\sin ^{3} \theta_{0}\left(1-\cos \left(\alpha-\alpha^{\prime}\right)\right)},
$$

and

$$
O\left(\epsilon_{\varphi}-\epsilon_{\varphi}^{\prime}\right): 0
$$

Integrating these terms with respect to $\alpha^{\prime}$ from 0 to $2 \pi$, we obtain the first order expansion of the integrals in (7) and (8)

$$
\begin{aligned}
& -\frac{1}{4 \pi} \mathrm{PV} \int_{0}^{2 \pi} \frac{\sin \theta^{\prime} \sin \left(\varphi-\varphi^{\prime}\right)}{1-\cos \gamma} d \alpha^{\prime} \\
& \quad=\frac{1}{4 \pi \sin \theta_{0}} \mathrm{PV} \int_{0}^{2 \pi} \frac{\epsilon_{\varphi}-\epsilon_{\varphi}^{\prime}}{1-\cos \left(\alpha-\alpha^{\prime}\right)} d \alpha^{\prime}+O\left(\epsilon^{2}\right)
\end{aligned}
$$

and 


$$
\begin{gathered}
-\frac{1}{4 \pi} \mathrm{PV} \int_{0}^{2 \pi} \frac{\cos \theta \sin \theta^{\prime} \cos \left(\varphi-\varphi^{\prime}\right)-\sin \theta \cos \theta^{\prime}}{\sin \theta(1-\cos \gamma)} d \alpha^{\prime} \\
=\frac{\cos \theta_{0}}{2 \sin ^{2} \theta_{0}}-\epsilon_{\theta} \frac{1+\cos ^{2} \theta_{0}}{2 \sin ^{3} \theta_{0}}+\frac{1}{4 \pi \sin ^{3} \theta_{0}} \\
\quad \times \mathrm{PV} \int_{0}^{2 \pi} \frac{\epsilon_{\theta}-\epsilon_{\theta}^{\prime}}{1-\cos \left(\alpha-\alpha^{\prime}\right)} d \alpha^{\prime}+O\left(\epsilon^{2}\right) .
\end{gathered}
$$

On the other hand, the flow induced by the pole vortices becomes

$$
\begin{aligned}
\frac{1}{4 \pi}( & \left.\frac{\Gamma_{1}}{1-\cos \left(\theta_{0}+\epsilon_{\theta}\right)}-\frac{\Gamma_{2}}{1+\cos \left(\theta_{0}+\epsilon_{\theta}\right)}\right) \\
= & V_{0}-\frac{\cos \theta_{0}}{2 \sin ^{2} \theta_{0}}-\epsilon_{\theta}\left(\frac{\left(\Gamma_{1}+\Gamma_{2}\right)\left(1+\cos ^{2} \theta_{0}\right)}{4 \pi \sin ^{3} \theta_{0}}\right. \\
& \left.+\frac{\left(\Gamma_{1}-\Gamma_{2}\right) \cos \theta_{0}}{2 \pi \sin ^{3} \theta_{0}}\right)+O\left(\epsilon^{2}\right) .
\end{aligned}
$$

Now, provided the small disturbances $\epsilon_{\theta}$ and $\epsilon_{\varphi}$ are represented by the following Fourier series:

$$
\begin{aligned}
& \epsilon_{\theta}(\alpha, t)=\sum_{n=-\infty}^{\infty} \theta_{n}(t) \exp (\text { in } \alpha), \\
& \epsilon_{\varphi}(\alpha, t)=\sum_{n=-\infty}^{\infty} \varphi_{n}(t) \exp (\text { in } \alpha) .
\end{aligned}
$$

Then, we have the linearized equations for the Fourier coefficients $\theta_{n}(t)$ and $\varphi_{n}(t)$

$$
\begin{aligned}
\frac{d \theta_{n}}{d t}= & \frac{|n|}{2 \sin \theta_{0}} \varphi_{n}, \\
\frac{d \varphi_{n}}{d t}= & \left(\frac{|n|}{2 \sin ^{3} \theta_{0}}-\frac{\left(\Gamma_{1}+\Gamma_{2}+2 \pi\right)\left(1+\cos ^{2} \theta_{0}\right)}{4 \pi \sin ^{3} \theta_{0}}\right. \\
& \left.-\frac{\left(\Gamma_{1}-\Gamma_{2}\right) \cos \theta_{0}}{2 \pi \sin ^{3} \theta_{0}}\right) \theta_{n},
\end{aligned}
$$

in which we use the following integral:

$$
\frac{1}{4 \pi} \mathrm{PV} \int_{0}^{2 \pi} \frac{1-\exp \left(i n\left(\alpha^{\prime}-\alpha\right)\right)}{1-\cos \left(\alpha-\alpha^{\prime}\right)} d \alpha^{\prime}=\frac{|n|}{2} \text {. }
$$

Thus the eigenvalues $\lambda_{n}$ of the linearized equations are given by

$$
\lambda_{n}= \pm \frac{1}{2 \sin ^{2} \theta_{0}} \sqrt{\left(|n|-\kappa_{1}\left(1+\cos ^{2} \theta_{0}\right)-\kappa_{2} \cos \theta_{0}\right)|n|},
$$

where

$$
\kappa_{1}=\frac{\Gamma_{1}+\Gamma_{2}+2 \pi}{2 \pi}
$$

and

$$
\kappa_{2}=\frac{\Gamma_{1}-\Gamma_{2}}{\pi} .
$$

Therefore, if the mode $n$ satisfies

$$
|n|<\kappa_{1}\left(1+\cos ^{2} \theta_{0}\right)+\kappa_{2} \cos \theta_{0},
$$

then the Fourier coefficients $\theta_{n}(t)$ and $\varphi_{n}(t)$ become neutrally stable. On the other hand, for sufficiently large $n$, since the positive eigenvalue approaches asymptotically to $|n| / 2 \sin ^{2} \theta_{0}$, a disturbance of high wave number grows like Kelvin-Helmholtz instability for planar flow.

We apply this stability condition to two special cases. First, when the strengths of both pole vortices are identical, namely $\kappa_{2}=0$, the stability condition is reduced to

$$
|n|<\kappa_{1}\left(1+\cos ^{2} \theta_{0}\right) \text {. }
$$

It indicates that when there is no pole vortex on the sphere, i.e., $\kappa_{1}=1$, the first spectra $\theta_{1}(t)$ and $\varphi_{1}(t)$ of disturbance are neutrally stable for arbitrary $\theta_{0} \neq \pi / 2$. On the other hand, for fixed $\kappa_{1}$, the number of stable spectra increases as the vortex sheet approaches either pole, i.e., $\theta_{0} \rightarrow 0$ or $\pi$.

Next, when the total vorticity on the sphere is zero, namely $\Gamma_{1}+\Gamma_{2}=-2 \pi$, the stability condition becomes

$$
|n|<\kappa_{2} \cos \theta_{0} \text {. }
$$

This means if the strength of the north pole vortex is greater than that of the south pole vortex, i.e., $\Gamma_{1}>\Gamma_{2}$, the vortex sheet in the northern hemisphere region has some neutrally stable spectra, while the vortex sheet in the southern hemisphere region has no stable spectra. Therefore, the vortex sheets in the northern hemisphere region evolve more stably than those in the southern hemisphere region at the initial moment of their evolution.

In what follows, we verify numerically the stability of the vortex sheet on the line of latitude, $\theta_{0}=\pi / 3$, for $\Gamma_{1}$ $+\Gamma_{2}=-2 \pi$. The criterion for the stability (13) is equivalent to

$$
|n|<\frac{\kappa_{2}}{2} .
$$

For each $n$, we compute the evolutions of $\theta_{n}(t)$ and $\varphi_{n}(t)$ for the following initial condition:

$$
\begin{aligned}
& \theta(\alpha, 0)=\frac{\pi}{3}+0.01 \frac{A_{n}}{\sqrt{A_{n}^{2}+B_{n}^{2}}} \sin n \alpha, \\
& \varphi(\alpha, 0)=\alpha+0.01 \frac{B_{n}}{\sqrt{A_{n}^{2}+B_{n}^{2}}} \sin n \alpha,
\end{aligned}
$$

where $\left(A_{n}, B_{n}\right)$ is an eigenvector corresponding to the positive eigenvalue of the linear equations (11) and (12). As for detailed numerical methods, see Sec. IV. Figure 1 shows the $\log$ plot of $\left|\theta_{n}(t)\right|$ and $\left|\varphi_{n}(t)\right|$ for $n=1,2,3,4$, and 5 from $t=0$ to 0.7 , when $\Gamma_{1}=1.5 \pi$ and $\Gamma_{2}=-3.5 \pi$. The first and the second spectra are neutrally stable, while the others grow exponentially in time. This numerical result agrees with the stability analysis.

\section{SINGULARITY FORMATION}

\section{A. Numerical methods}

We explain the numerical methods used here to compute the evolution of the vortex sheet. There are two difficulties in the numerical computation. First, the linear stability analysis in Sec. III indicates that Eqs. (7) and (8) are ill-posed in the 

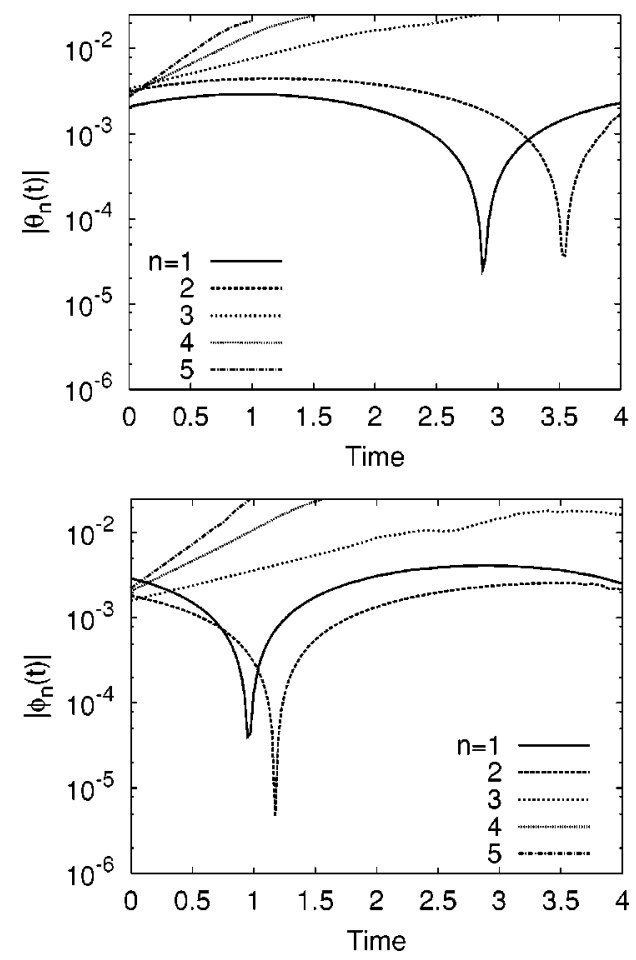

FIG. 1. Log plot of $\left|\theta_{n}(t)\right|$ and $\left|\varphi_{n}(t)\right|$ for $n=1,2,3,4$, and 5 from $t=0$ to 0.7. The strengths of pole vortices are $\Gamma_{1}=1.5 \pi$ and $\Gamma_{2}=-3.5 \pi$.

sense of Hadamard, because a disturbance of high wave number $n$ grows exponentially at a rate proportional to $|n|$. Consequently, a small round-off error grows rapidly due to this instability. Second, since the integrals in the equation are singular, the trapezoidal rule quadrature fails to compute them accurately. In order to alleviate these drawbacks, we implement the spectral filtering technique by Krasny ${ }^{5}$ and the alternate point quadrature by Sidi and Israeli. ${ }^{19}$

In the spectral filtering technique, if the absolute value of a spectrum of disturbance is less than a prescribed small threshold, we adjust it to zero. It enables us to eliminate spurious instability due to round-off error. As a matter of fact, Caflisch et al. ${ }^{20}$ showed that this technique gives an optimal numerical approximation for the singularity formation. In addition, it has been successfully applied to ill-posed vortex sheet problems. ${ }^{5,6,21-23}$

On the other hand, the alternate point quadrature gives an accurate approximation for the integration of weakly singular periodic functions and has been implemented successfully in other vortex sheet problems. ${ }^{6,10,23}$ It is applied to the present problem in the following way. We discretize the interval $\alpha \in[0,2 \pi)$ by $N$ segments and put $h=2 \pi / N$ and $\alpha_{i}$ $=i h$. Let $f\left(\alpha, \alpha^{\prime}\right)$ denote the integrand in Eq. (7). Then, the singular integral at $\alpha=\alpha_{i}$ is approximated by

$$
\mathrm{PV} \int_{0}^{2 \pi} f\left(\alpha_{i}, \alpha^{\prime}\right) d \alpha^{\prime} \approx 2 h \sum_{k: k+i=o d d} f\left(\alpha_{i}, \alpha_{k}\right) .
$$

This quadrature also gives an accurate approximation for the singular integral in (8).

In order to estimate the singularity formation, we use the spectral fitting method developed by Sulem et al. ${ }^{24}$ Assume

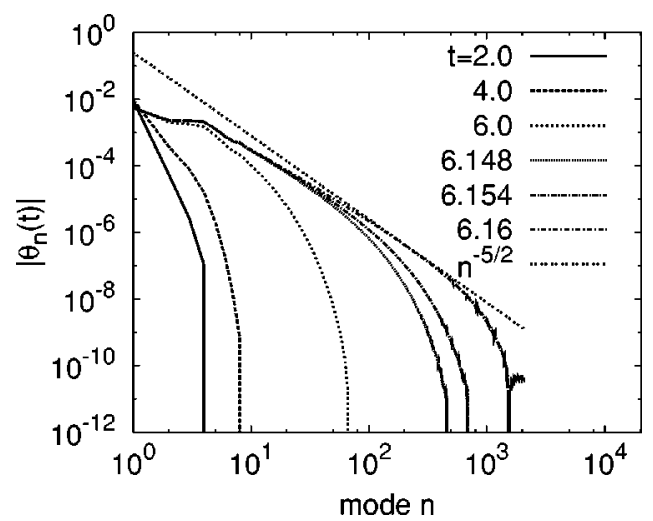

FIG. 2. $\log -\log$ plot of the Fourier coefficients in the $\theta$ variable vs mode $n$ from $t=2$ to 6.16 , when $\theta_{0}=\pi / 3$ and $\Gamma_{1}=\Gamma_{2}=0$. The high spectra approach to the power-law slope $n^{-2.5}$.

that the Fourier coefficients in (9) and (10) have the following asymptotic forms for $t \gg 1$ :

$$
\begin{aligned}
& \left|\theta_{n}(t)\right| \sim C_{\theta} n^{-p_{\theta}} \exp \left(-\delta_{\theta} n\right), \\
& \left|\varphi_{n}(t)\right| \sim C_{\varphi} n^{-p_{\varphi}} \exp \left(-\delta_{\varphi} n\right),
\end{aligned}
$$

in which the parameters $C_{\theta}, C_{\varphi}, p_{\theta}, p_{\varphi}, \delta_{\theta}$, and $\delta_{\varphi}$ are independent of the wave number $n$. Note that the parameters $\delta_{\theta}$ and $\delta_{\varphi}$ determine analyticity of $\theta$ and $\varphi$, while $p_{\theta}$ and $p_{\varphi}$ give the order of the singularity for each variable. We compute these parameters from the numerical data by a leastsquare fit. Then, we estimate times when $\delta_{\theta}$ and $\delta_{\varphi}$ vanish by extrapolation, at which the variables $\theta$ and $\varphi$ lose their smoothness. This method also has been successfully applied to many numerical studies of singularity formation in vortex sheet motion. ${ }^{5,6,22,23,25}$

We consider the following initial condition of the vortex sheet:

$$
\begin{aligned}
& \theta(\alpha, 0)=\theta_{0}+0.01 \sin \alpha, \\
& \varphi(\alpha, 0)=\alpha+0.01 \sin \alpha,
\end{aligned}
$$

in which $\theta_{0}$ is a steady state. As for temporal integration, we use the fourth-order Runge-Kutta scheme. We implement the filtering technique every time step. The filtering threshold is $K=1.0 \times 10^{-11}$, since we perform 64-bit computation. The vortex sheet is discretized by $N=4096$ points and the step size for the Runge-Kutta method is $\Delta t=1.0 \times 10^{-3}$.

\section{B. Stability and singularity formation}

Figure 2 shows the $\log -\log$ plot of the Fourier coefficients $\left|\theta_{n}(t)\right|$ in (9) from $t=2$ to 6.16 for $\Gamma_{1}=\Gamma_{2}=0$. The high spectra grow rapidly and tend to the power-law line $n^{-2.5}$ near $t=6.16$, which indicates that the vortex sheet acquires the curvature singularity that is frequently observed in 2D vortex-sheet problems. To confirm this observation, we compute the parameters $\delta_{\theta}$ and $\delta_{\varphi}$ by the spectral fitting method. Figure 3 shows the plot of these parameters from $t$ $=6$ to 6.16 . They decrease monotonically towards zero. Estimating the times when $\delta_{\theta}(t)$ and $\delta_{\varphi}(t)$ vanish, we have $t_{\theta}^{*}=t_{\varphi}^{*} \approx 6.17$, which indicates that the variables $\theta$ and $\varphi$ lose their analyticity at the same time. We remark that the present 


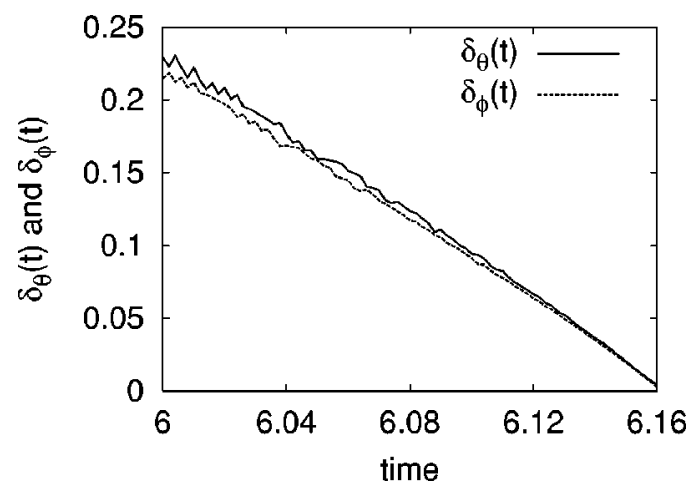

FIG. 3. Plot of $\delta_{\theta}(t)$ and $\delta_{\varphi}(t)$ from $t=6.0$ to 6.16 that determine the analyticity of the $\theta$ and $\varphi$ variables. The strengths of the pole vortices are $\Gamma_{1}=0$ and $\Gamma_{2}=0$. They decrease linearly to zero at the same time. The singularity appears when they vanish.

numerical computation is too inaccurate to determine the precise values of the indices $p_{\theta}$ and $p_{\varphi}$, although Fig. 2 indicates the curvature singularity. As we can see in Ref. 6, more resolution with high machine precision is required to determine these indices.

The linear stability analysis shows that the initial disturbances $\theta_{1}(t)$ and $\varphi_{1}(t)$ are neutrally stable when $\Gamma_{1}=\Gamma_{2}$ $=0$. Nevertheless, the singularity eventually appears. We give a reason for this phenomenon. Figure 4 shows the log plot of $\left|\theta_{n}(t)\right|$ from $n=1$ to 10 up to the singularity time. While the spectrum $\theta_{1}(t)$ remains neutrally stable due to the linear stability, the unstable spectra arise one by one due to nonlinearity of the equation and then grow exponentially because of their own instability, which leads to the singularity formation.

Now, we focus on how the change of stability affects the singularity formation. First, we fix the stationary vortex sheet at $\theta_{0}=\pi / 3$ and change the strengths of the pole vortices. Figure 5 is the $\log -\log$ plot of the Fourier coefficients $\left|\theta_{n}(t)\right|$ from $t=2$ to 7.743 for $\Gamma_{1}=1.5 \pi$ and $\Gamma_{2}=-3.5 \pi$, in which $\left|\theta_{1}(t)\right|$ and $\left|\theta_{2}(t)\right|$ are neutrally stable. The high spectra approach to the power-law line $n^{-2.5}$ as time approaches to $t \approx 7.745$, which also indicates that the curvature singular-

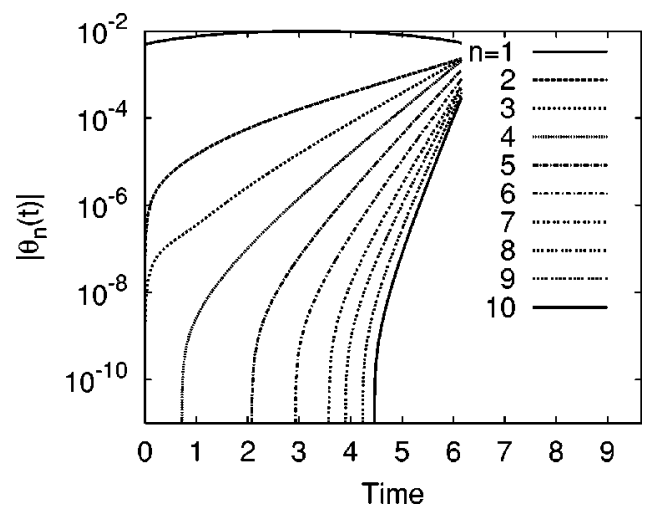

FIG. 4. Log plot of $\left|\theta_{n}(t)\right|$ from $n=1$ to 10 up to the singularity formation. The strengths of the pole vortices are $\Gamma_{1}=0$ and $\Gamma_{2}=0$. The spectrum $\theta_{1}(t)$ remains stable. However, the high spectra are induced one by one due to nonlinearity, and they grow exponentially afterwards because of their own linear instability.

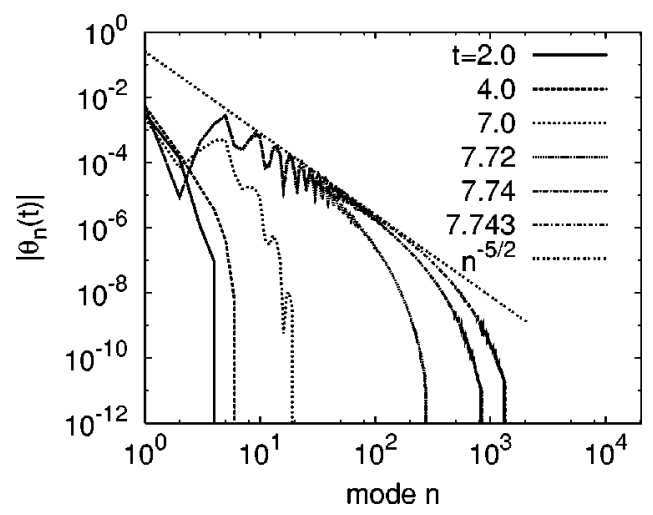

FIG. 5. $\log -\log$ plot of the Fourier coefficients in the $\theta$ variable vs mode $n$ from $t=2$ to 7.743 , when $\theta_{0}=\pi / 3, \Gamma_{1}=1.5 \pi$, and $\Gamma_{2}=-3.5 \pi$. The high spectra approach to the power-law slope $n^{-2.5}$.

ity appears. However, the singularity develops later compared to the previous case in which there is no pole vortex.

We give a reason for the delay of the singularity formation. Figure 6 shows the log plot of $\left|\theta_{n}(t)\right|$ from $n=1$ to 10 up to the singularity time. The spectra $\theta_{1}(t)$ and $\theta_{2}(t)$ remain neutrally stable due to the linear stability. Since the number of neutrally stable modes increases, owing to the pole vortices, it takes a longer time until the unstable higher modes are induced by nonlinearity of the equation, which results in the delay of the singularity formation.

Next we compute times of the singularity formation for various $\theta_{0}, \Gamma_{1}$, and $\Gamma_{2}$. Figure 7 shows the singularity times when $\Gamma_{1}=\Gamma_{2}=-\pi, \Gamma_{1}=\Gamma_{2}=0$, and $\Gamma_{1}=\Gamma_{2}=\pi$. According to the stability condition (14), all modes are unstable in the first case, the first spectra $\theta_{1}(t)$ and $\varphi_{1}(t)$ are neutrally stable in the second case, and the first and the second spectra are neutrally stable in the last case. While the vortex sheet acquires the curvature singularity in all cases, it develops later as the number of stable modes increases. This is because the destabilization of the vortex sheet is delayed due to the increase of stable low spectra. On the other hand, however, the existence time of the vortex sheet approaches zero as $\theta_{0}$ tends to the poles, because the motion of the vortex sheet becomes singular, i.e., $V_{0} \rightarrow \infty$, as $\theta_{0} \rightarrow 0$ and $\pi$ regardless of the linear stability.

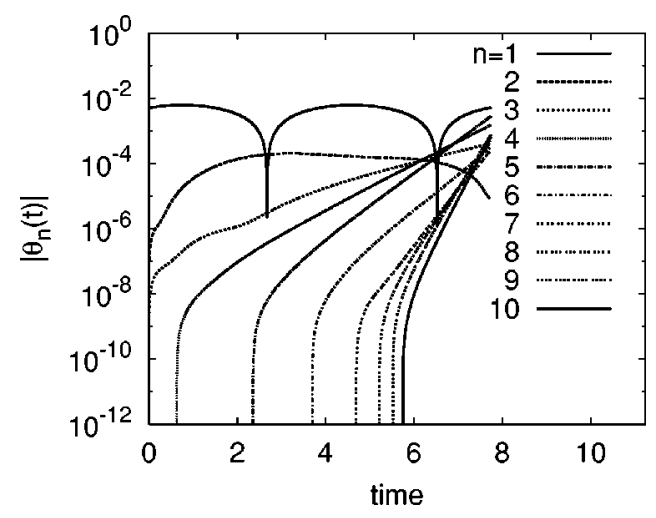

FIG. 6. Log plot of $\left|\theta_{n}(t)\right|$ from $n=1$ to 10 up to the singularity formation. The strengths of the pole vortices are $\Gamma_{1}=1.5 \pi$ and $\Gamma_{2}=-3.5 \pi$. The spectra $\theta_{1}(t)$ and $\theta_{2}(t)$ remain stable, but the high spectra grow exponentially. 


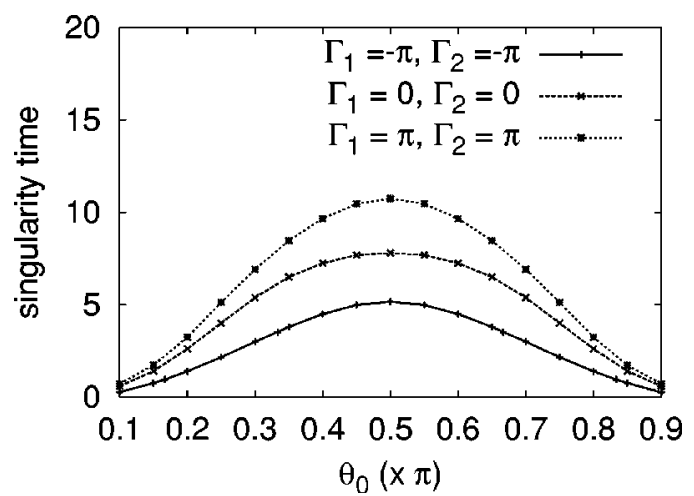

FIG. 7. Singularity times of the vortex sheets for various $\theta_{0}$ when $\Gamma_{1}=\Gamma_{2}$ $=0$ and $\pm \pi$. As the summation $\Gamma_{1}+\Gamma_{2}$ increases, the vortex sheet remains smooth longer for all $\theta_{0}$.

We plot the singularity times for another case when $\Gamma_{1}$ $>\Gamma_{2}$ with $\Gamma_{1}+\Gamma_{2}=-2 \pi$ in Fig. 8. The vortex sheets in the northern hemisphere region remain smooth longer than those in the southern hemisphere region as the difference $\Gamma_{1}-\Gamma_{2}$ increases. It is closely related to the linear stability result (15); the vortex sheet in the northern hemisphere region has more linearly stable low spectra than that in the southern hemisphere region.

\section{LONG TIME EVOLUTION}

Because of the singularity formation, it is impossible to compute evolution of vortex sheet beyond the critical time. In the same way as in the computation of $2 \mathrm{D}$ vortex sheets, introducing an artificial parameter $\sigma$, we consider the following regularized equation:

$$
\theta_{t}=-\frac{1}{4 \pi} \mathrm{PV} \int_{0}^{2 \pi} \frac{\sin \theta^{\prime} \sin \left(\varphi-\varphi^{\prime}\right)}{1-\cos \gamma+\sigma^{2}} d \alpha^{\prime},
$$

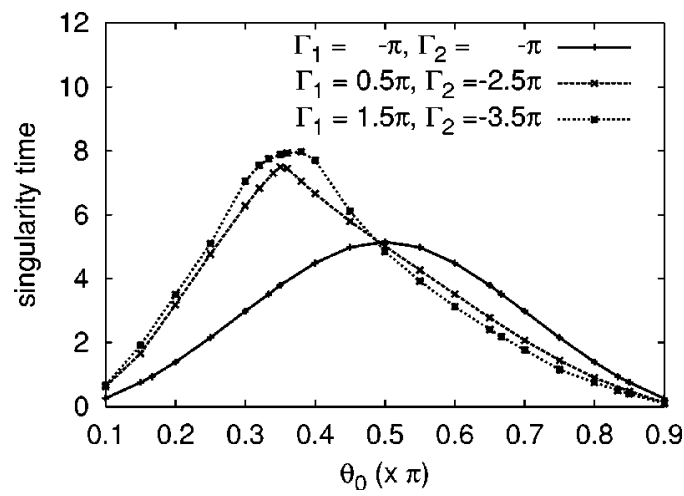

FIG. 8. Singularity times of the vortex sheets for various $\theta_{0}, \Gamma_{1}$, and $\Gamma_{2}$ with $\Gamma_{1}+\Gamma_{2}=-2 \pi$. When $\Gamma_{1}>\Gamma_{2}$, the vortex sheets in the northern hemisphere regions remain smooth longer than those in the southern hemisphere regions. As the difference in the strengths of the pole vortices increases, the vortex sheet blows up later due to the linear stability.

$$
\begin{aligned}
\varphi_{t}= & -\frac{1}{4 \pi} \\
& \times \mathrm{PV} \int_{0}^{2 \pi} \frac{\cos \theta \sin \theta^{\prime} \cos \left(\varphi-\varphi^{\prime}\right)-\sin \theta \cos \theta^{\prime}}{\sin \theta\left(1-\cos \gamma+\sigma^{2}\right)} d \alpha^{\prime} \\
& +\frac{1}{4 \pi}\left(\frac{\Gamma_{1}}{1-\cos \theta}-\frac{\Gamma_{2}}{1+\cos \theta}\right)
\end{aligned}
$$

The regularization parameter is fixed to $\sigma=0.1$. The initial condition and the other numerical parameters are the same as those in Sec. IV.

Figure 9 shows the evolution of the vortex sheet at $\theta_{0}$ $=\pi / 3$ from $t=13$ to 19 for $\Gamma_{1}=\Gamma_{2}=0$. Let us recall that the initial disturbances $\theta_{1}(t)$ and $\varphi_{1}(t)$ are linearly stable in this case. Then, the vortex sheet begins rolling up and finally develops into a structure with four rolling-up spirals. On the other hand, Fig. 10 shows snapshots of the solution for $\Gamma_{1}$ $=1.5 \pi$ and $\Gamma_{2}=-3.5 \pi$, in which $\theta_{n}(t)$ and $\varphi_{n}(t)$ for $n$ $=1$ and 2 are stable. While the vortex sheet evolves stably up to $t=17$, five rolling-up spirals appear after $t=21$. We also plot the evolution for $\left(\Gamma_{1}, \Gamma_{2}\right)=(-\pi,-\pi)$, in which all modes are unstable in Fig. 11, and the evolution for $\left(\Gamma_{1}, \Gamma_{2}\right)=(2.5 \pi,-4.5 \pi)$, in which $\theta_{n}(t)$ and $\varphi_{n}(t)$ for $n$ $=1,2$, and 3 are stable in Fig. 12. These evolutions show that as the number of stable low spectra increases, the vortex sheet tends to remain stable for longer time, but it eventually evolves into a complex structure with more rolling-up spirals.

We observe this phenomenon from a spectral point of view. Figure 13 shows the evolution of the Fourier coefficients $\theta_{n}(t)$ for $n=1, \ldots, 10$ when $\Gamma_{1}=1.5 \pi$ and $\Gamma_{2}$ $=-3.5 \pi$. The initial spectrum $\theta_{1}(t)$ is neutrally stable and the other spectra are sufficiently small until $t=15$. Hence, the vortex sheet evolves stably up to this time. After $t=15$, while $\theta_{1}(t)$ and $\theta_{2}(t)$ remain small because of the linear stability, some of the unstable high spectra grow large, which leads to the rolling-up of the vortex sheet. The Fourier coefficients $\theta_{5}(t)$ and $\theta_{10}(t)$ are dominant spectra in the solution after $t=20$, which indicates that the five spirals emerge. Thus, as the number of stable low spectra increases, owing to the pole vortices, the vortex sheet evolves stably for a longer time. However, once the unstable spectra arise and grow large, the vortex sheet rolls up and develops into many rolling-up spirals. Moreover, since the number of spirals is determined by the dominant unstable high spectra in the solution, the vortex sheet tends to have more spirals as the linearly stable low spectra increases.

Finally, we note that the roll-up structure depends on the regularization parameter $\sigma$. Figure 14 shows the roll-up structure of the vortex sheet after long time evolution for various $\sigma$. Each solution has five spirals, while these spirals have more winding as $\sigma$ tends to zero. Thus the solution is likely to converge to five rolling-up spirals with infinitely tight winding when $\sigma \rightarrow 0$. However, when $\sigma$ is too small, the evolution is quite unstable numerically and many small-scale rolling-up spirals appear in the numerical solution due to insufficient accuracy. Hence, many discretizing points and 


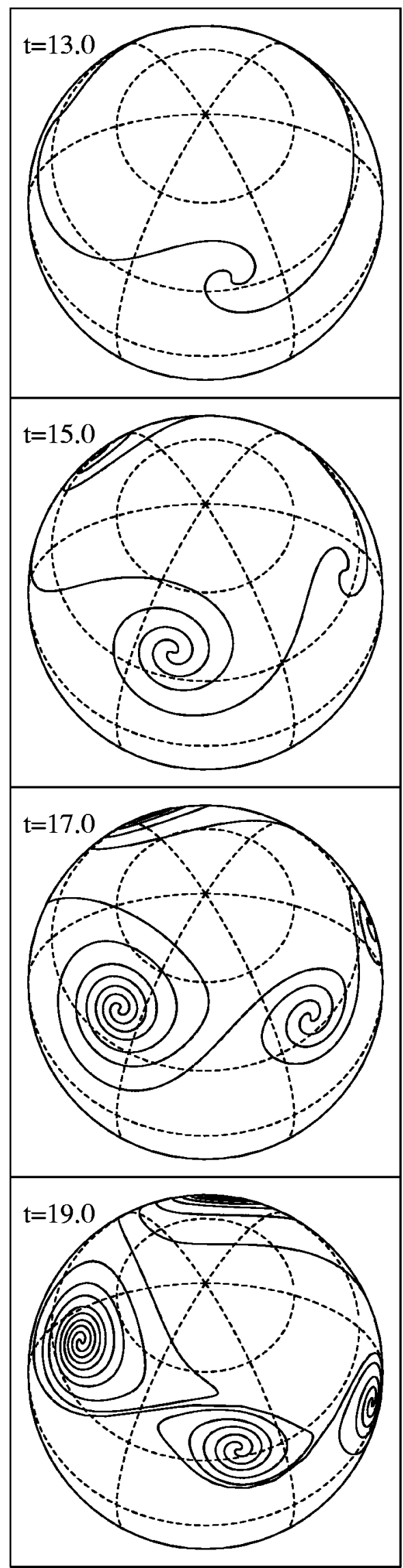

FIG. 9. Evolution of the vortex sheet at $\theta_{0}=\pi / 3$ from $t=13$ to 19 when $\Gamma_{1}=0$ and $\Gamma_{2}=0$, in which the first spectra of disturbance are linearly stable. Four spirals moving westward appear.

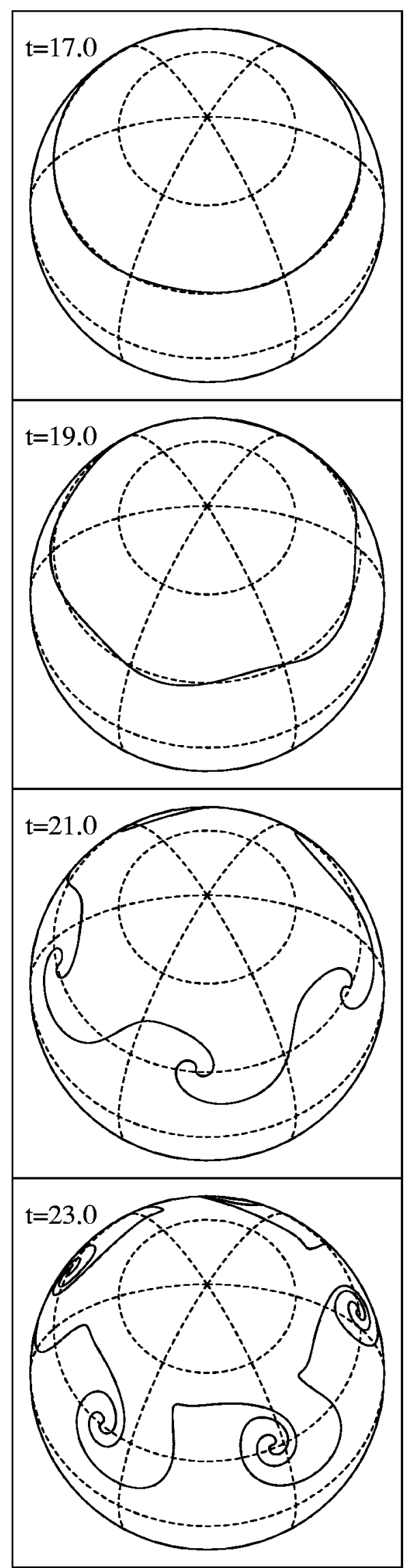

FIG. 10. Evolution of the vortex sheet at $\theta_{0}=\pi / 3$ from $t=17$ to 23 when $\Gamma_{1}=1.5 \pi$ and $\Gamma_{2}=-3.5 \pi$, in which the first and the second spectra are linearly stable. Five spirals moving westward appear. 


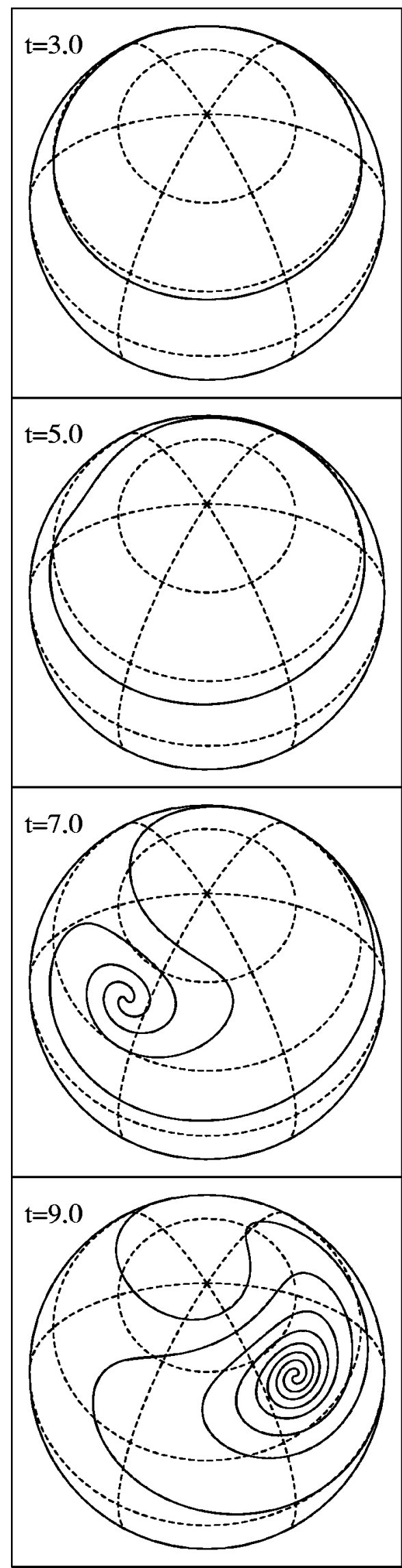

FIG. 11. Evolution of the vortex sheet at $\theta_{0}=\pi / 3$ from $t=3$ to 9 when $\Gamma_{1}=-\pi$ and $\Gamma_{2}=-\pi$, in which all spectra are unstable. One big rolling-up spiral appears moving westward.

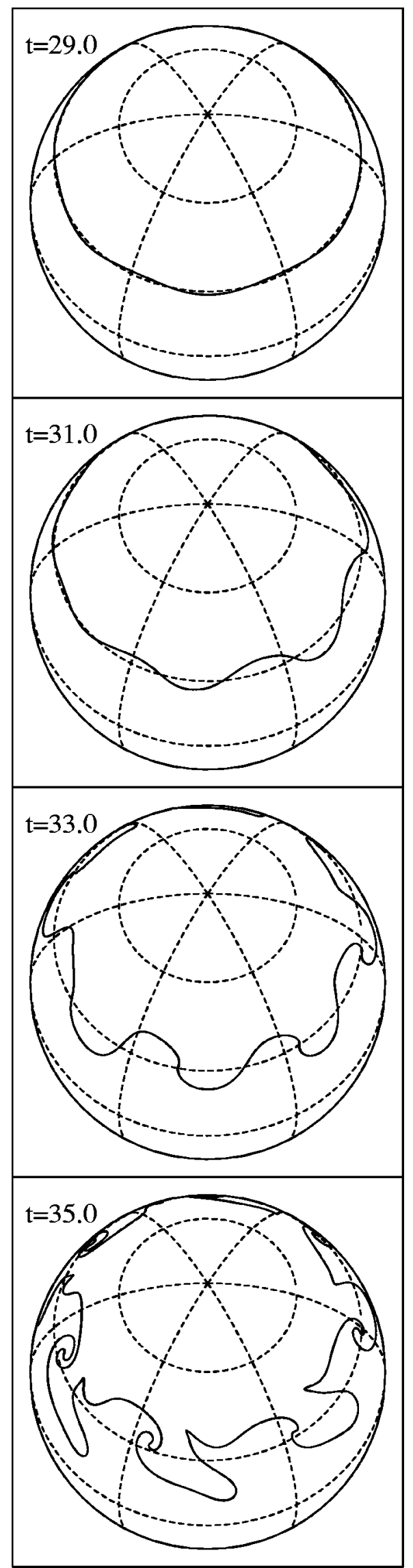

FIG. 12. Evolution of the vortex sheet at $\theta_{0}=\pi / 3$ from $t=29$ to 35 when $\Gamma_{1}=2.5 \pi$ and $\Gamma_{2}=-4.5 \pi$, in which the first, the second, and the third spectra are linearly stable. Seven rolling-up spirals appear moving westward. 


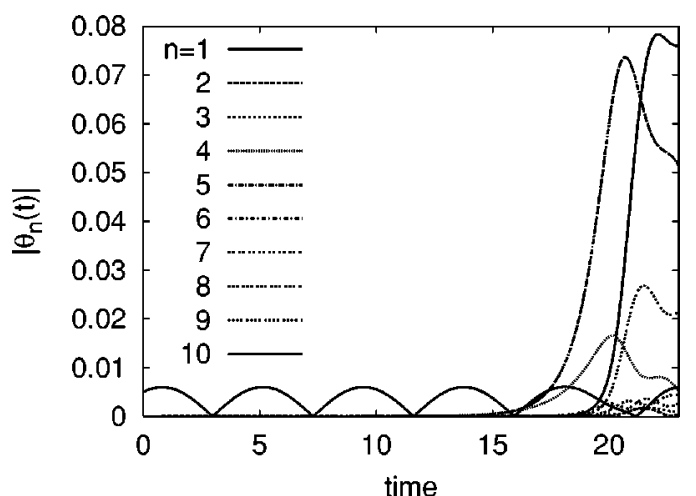

FIG. 13. Evolution of the spectra $\left|\theta_{n}(t)\right|$ for $n=1, \ldots, 10$ up to $t=23$ when $\Gamma_{1}=1.5 \pi$ and $\Gamma_{2}=-3.5 \pi$. While the spectra $\theta_{1}(t)$ and $\theta_{2}(t)$ are neutrally stable, the other spectra are unstable and grow rapidly. The Fourier coefficients $\theta_{5}(t)$ and $\theta_{10}(t)$ are dominant in the solution, which results in the appearance of the five rolling-up spirals in Fig. 10.

higher precision arithmetic are required to verify the convergence of the solution when $\sigma \rightarrow 0$.

\section{CONCLUSION AND DISCUSSION}

We have considered the motion of a single vortex sheet on the surface of a unit sphere with fixed point vortices at the poles. First, we derive the equation for the vortex sheet on the sphere from the equations for $N$ point vortices.

Second, we have investigated the linear stability for the steady solution located at a line of latitude. When there is no pole vortex on a sphere, the first spectra $\theta_{1}(t)$ and $\varphi_{1}(t)$ become neutrally stable. This is an effect of the curvature of the sphere on the linear stability. Furthermore, depending on the position of the stationary vortex sheet and the strengths of pole vortices, it is possible to stabilize more low spectra or to destabilize all spectra. On the other hand, for sufficiently large $n$, the Fourier spectra $\theta_{n}(t)$ and $\varphi_{n}(t)$ become inevitably unstable and their exponentially growing rate approach asymptotically to $\lambda_{n} \sim|n| / 2 \sin ^{2} \theta_{0}$, which is the same as Kelvin-Helmholtz instability for planar flow. A similar stability result is observed in the study of a $2 \mathrm{D}$ vortex sheet in the presence of background shear flow. ${ }^{13,26}$

Third, although some low spectra in the solution are linearly stable, the vortex sheet itself evolves unstably; we verify numerically that the curvature singularity appears in the vortex sheet in finite time regardless of the linear stability, while the appearance of the singularity is getting later as the vortex sheet has more stable spectra. Numerical computation of the equations regularized by the vortex method shows that the vortex sheet develops into more rolling-up spirals as it has more linearly stable spectra, although it evolves stably for a longer time owing to the stability of the low spectra. These phenomena are largely due to the rapid growth of the high spectra that the linear stability is unable to control.

Last, we discuss a connection between the vortex sheet and a band of constant vorticity on the sphere. Generally speaking, the vortex sheet is derived from the band of vorticity when its thickness tends to zero, keeping the circulation fixed. In fact, Benedetto and Pulvirenti ${ }^{27}$ showed that 2D

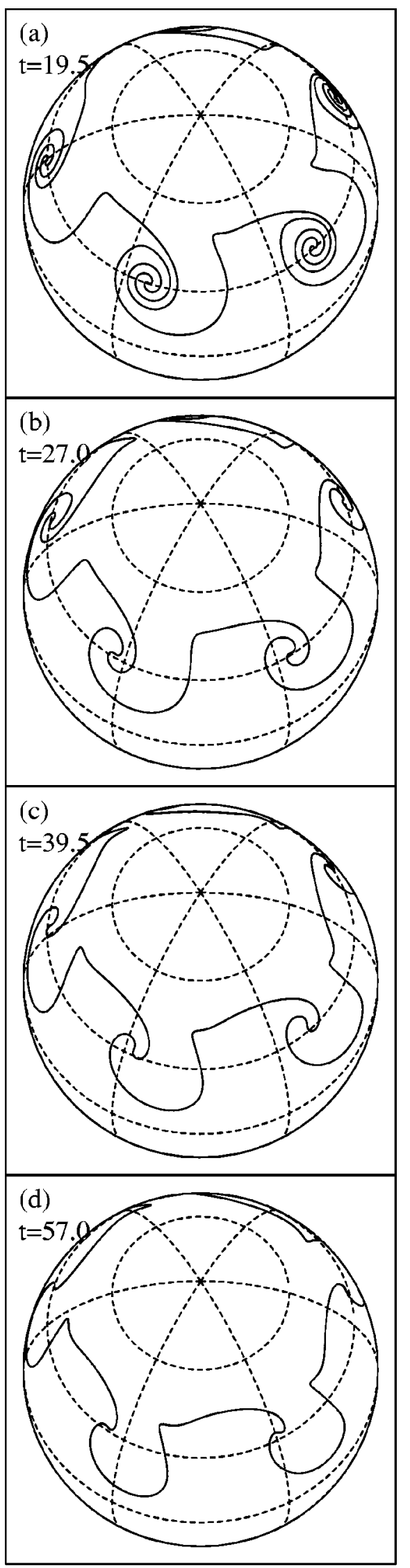

FIG. 14. Roll-up structure of the vortex sheet for various regularization parameters $\sigma$ : (a) $\sigma=0.08$, (b) $\sigma=0.12$, (c) $\sigma=0.16$, and (d) $\sigma=0.2$. The strengths of the pole vortices are $\Gamma_{1}=1.5 \pi$ and $\Gamma_{2}=-3.5 \pi$. As $\sigma$ tends to zero, the vortex sheet has five spirals with more winding. 
vortex layers converge uniformly to $2 \mathrm{D}$ vortex sheets insofar as both of them are sufficiently smooth. Dritschel and Polvani ${ }^{16}$ investigated stability of a vortex strip in which the vorticity is constant, on the sphere with the pole point vortices. They indicated that a thinner vortex strip tends to be more unstable and the vortex strips in the northern hemisphere are more stable than those in the southern hemisphere when the strength of the point vortex at the north pole is larger than that at the south pole. In addition, they also concluded that the presence of the pole vortices fails to eliminate the instability. These results are similar to our stability results. Hence, the vortex strips connect with the vortex sheet continuously in terms of the stability. On the other hand, since the vortex sheet acquires the curvature singularity, the continuity never holds after the singularity time. In particular, internal structure of the rolling-up spiral in the vortex sheet is different from that of the vortex strip, as we observed in Krasny ${ }^{7}$ and Baker and Shelley. ${ }^{28}$

\section{ACKNOWLEDGMENT}

This research is partially supported by Ministry of Education, Science, Sports and Culture, Grand-in-Aid for Young Scientists (B), Grant No. 14740069, 2003.

${ }^{1}$ P. G. Saffman, Vortex Dynamics (Cambridge University Press, Cambridge, 1992).

${ }^{2}$ G. Birkhoff, "Helmholtz and Taylor instability," Proc. Symp. Appl. Math. 13, 55 (1962).

${ }^{3}$ D. W. Moore, "The spontaneous appearance of a singularity in the shape of an evolving vortex sheet," Proc. R. Soc. London, Ser. A 365, 105 (1979).

${ }^{4}$ S. J. Cowley, G. R. Baker, and S. Tanveer, "On the formation of Moore curvature singularities in vortex sheets," J. Fluid Mech. 378, 233 (1999).

${ }^{5} \mathrm{R}$. Krasny, "A study of singularity formation in a vortex sheet by the point-vortex approximation," J. Fluid Mech. 167, 65 (1986).

${ }^{6}$ M. J. Shelley, "A study of singularity formation in vortex-sheet motion by a spectrally accurate vortex method," J. Fluid Mech. 244, 493 (1992).

${ }^{7}$ R. Krasny, "Desingularization of periodic vortex sheet roll-up," J. Comput. Phys. 65, 292 (1986).
${ }^{8}$ R. E. Caflisch and J. Lowengrub, "Convergence of the vortex method for vortex sheets," SIAM J. (Soc. Ind. Appl. Math) Numer. Anal. 26, 1060 (1989).

${ }^{9}$ A. J. Majda, "Remarks on weak solutions for vortex sheets with a distinguished sing," Indiana Univ. Math. J. 42, 921 (1993).

${ }^{10}$ R. E. Caflisch, X. Li, and M. J. Shelley, "The collapse of an axisymmetric, swirling vortex sheet," Nonlinearity 6, 843 (1993).

${ }^{11}$ R. Krasny, "Computation of vortex sheet roll-up in the Trefftz plane," J. Fluid Mech. 184, 123 (1987).

${ }^{12} \mathrm{M}$. Nitsche and R. Krasny, "A numerical study of vortex ring formation at the edge of a circular tube," J. Fluid Mech. 276, 139 (1994).

${ }^{13}$ T. Sakajo and H. Okamoto, "Numerical computation of vortex sheet roll-up in the background shear flow," Fluid Dyn. Res. 17, 195 (1996).

${ }^{14} \mathrm{~T}$. Sakajo, "Numerical computation of a three dimensional vortex sheet in a swirl flow," Fluid Dyn. Res. 28, 423 (2001).

${ }^{15} \mathrm{P}$. K. Newton, The N-Vortex Problem, Analytic Techniques (Springer, New York, 2001).

${ }^{16}$ D. G. Dritschel and L. M. Polvani, "The roll-up of vorticity strips on the surface of a sphere," J. Fluid Mech. 234, 47 (1992).

${ }^{17}$ L. M. Polvani and D. G. Dritschel, "Wave and vortex dynamics on a sphere,” J. Fluid Mech. 255, 35 (1993).

${ }^{18}$ Y. Kimura and H. Okamoto, "Vortex motion on a sphere," J. Phys. Soc. Jpn. 56, 4203 (1987).

${ }^{19}$ A. Sidi and M. Israeli, "Quadrature methods for periodic singular and weakly singular Fredholm integral equations," J. Sci. Comput. 3, 201 (1988).

${ }^{20}$ R. E. Caflisch, T. Y. Hou, and J. Lowengrub, "Almost optimal convergence of the point vortex method for vortex sheets using numerical filtering," Math. Comput. 68, 1465 (1999).

${ }^{21}$ J. S. Ely and G. R. Baker, "High-precision calculations of vortex sheet motion," J. Comput. Phys. 111, 275 (1993).

${ }^{22} \mathrm{M}$. Nitsche, "Singularity formation in a cylindrical and a spherical vortex sheet," J. Comput. Phys. 173, 208 (2001).

${ }^{23} \mathrm{~T}$. Sakajo, "Formation of curvature singularity along vortex line in an axi-symmetric, swirling flow," Phys. Fluids 14, 2886 (2002).

${ }^{24}$ C. Sulem, P. L. Sulem, and H. Frisch, "Tracing complex singularities with spectral methods," J. Comput. Phys. 50, 138 (1983).

${ }^{25} \mathrm{Q}$. Nie and G. Baker, "Application of adaptive quadrature to axisymmetric vortex sheet motion," J. Comput. Phys. 143, 49 (1999).

${ }^{26}$ M. Kiya and M. Arie, "Helmholtz instability of a vortex sheet in uniform shear flow," Phys. Fluids 22, 378 (1979).

${ }^{27}$ D. Benedetto and M. Pulvirenti, "From vortex layers to vortex sheets," SIAM (Soc. Ind. Appl. Math.) J. Appl. Math. 52, 1041 (1992).

${ }^{28}$ G. R. Baker and M. J. Shelley, "On the connection between thin vortex layers and vortex sheets," J. Fluid Mech. 215, 161 (1990). 